\title{
Predictive optimal control of sewer networks using CORAL tool: application to Riera Blanca catchment in Barcelona
}

\author{
V. Puig, G. Cembrano, J. Romera, J. Quevedo, B. Aznar, G. Ramón \\ and J. Cabot
}

\begin{abstract}
This paper deals with the global control of the Riera Blanca catchment in the Barcelona sewer network using a predictive optimal control approach. This catchment has been modelled using a conceptual modelling approach based on decomposing the catchments in subcatchments and representing them as virtual tanks. This conceptual modelling approach allows real-time model calibration and control of the sewer network. The global control problem of the Riera Blanca catchment is solved using a optimal/predictive control algorithm. To implement the predictive optimal control of the Riera Blanca catchment, a software tool named CORAL is used. The on-line control is simulated by interfacing CORAL with a high fidelity simulator of sewer networks (MOUSE). CORAL interchanges readings from the limnimeters and gate commands with MOUSE as if it was connected with the real SCADA system. Finally, the global control results obtained using the predictive optimal control are presented and compared against the results obtained using current local control system. The results obtained using the global control are very satisfactory compared to those obtained using the local control.
\end{abstract}

Key words | decision support, optimal control, predictive control, real-time control, sewer systems

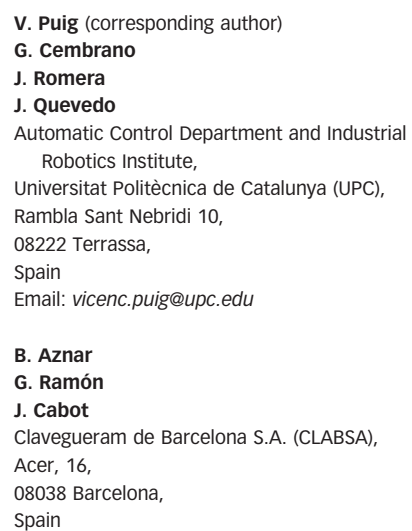

\section{INTRODUCTION}

Urban drainage systems are generally larges-scale networks of sewers which carry urban wastewater and rainwater to one or more terminal points, where it is treated before disposal to a receiving environment. Combined sewer systems carry rain- and wastewater together. In many cities that has been growing fast and stormy rains are frequent, the passive use of the infrastructure may be inefficient to convey all the rain- and wastewater to the treatment plants when high-intensity rain occurs. This results in flooding of certain areas and combined sewer overflows (CSO) which release untreated water to the environment.

Advanced urban drainage involves the incorporation of active control of the sewage systems (Price 2000; Schütze et al. 2004). On the one hand, detention tanks, with its associated inlet and outlet gates are installed, as well as other flow-control devices, such diversion gates, weirs and pumping stations. On the other, a telemetry and supervisory control system (SCADA) is required. The telemetry system contains rain-gauges distributed in several areas of the city, as well as flow or level meter (limnimeters) and quality meters in the main sewers, which periodically send information to a central dispatch. The supervisory control system allows operators to monitor the sewer network and command the flow-control elements. Detention tanks are used to store water during heavy rain and gradually release it when the sewage network is not overloaded. Flow-diversion 
and detention-tank gates must be actuated so as to reduce flooding and polluting discharges to the environment.

Real-time control of an urban drainage system may be local or global. When local control is applied, flow regulation devices use only measurements taken at its specific location. While this control structure is applicable in many simple cases, in a large city, with a strongly interconnected sewerage network and a complex network of actuators and sensors, it may not be the most efficient alternative. Conversely, global control, which computes control actions taking into account real-time measurements all through the network, is likely to make the best use of the infrastructure capacity and all the available sensor information. Global predictive/optimal control in urban drainage networks deals with the problem of generating control strategies for the control elements in a sewer network, ahead of time, based on a predictive dynamic model of the system, as well as readings of the telemetry system, in order to minimize flooding and CSO.

Optimal predictive control in water systems has been successfully applied to water supply and distribution in several applications (e.g. Brdys \& Ulanicki 1994; Cembrano et al. 2005). More recently, this type of control structure is being applied in the context of advanced urban drainage (e.g. Gelormino \& Ricker 1994; Pleau et al. 2005; OcampoMartínez et al. 2008).

This paper addresses the global control of the Riera Blanca catchment in the Barcelona sewer network using a predictive optimal control approach. This catchment has been modelled using a conceptual modelling approach based on decomposing the catchments in subcatchments and representing them as virtual tanks. This conceptual modelling approach allows real-time model calibration and control of the sewer network. The global control problem of the Riera Blanca catchment is solved using a optimal/predictive control algorithm. To implement predictive optimal control of the Riera Blanca catchment, a software tool named CORAL is used. The on-line control is simulated by interfacing CORAL with a high fidelity simulator of sewer networks (MOUSE). CORAL interchanges liminimeters readings and gate commands with MOUSE as if it was connected with the real SCADA system. The global control results obtained using the predictive optimal control will be compared with those obtained using current local control system.
The paper is organized in the following manner. First, the modelling principles used for the optimal/predictive control of sewer networks are presented. Then, the global control problem of a sewer network is addressed suing optimal/predictive control approach. The control implementation used is done using CORAL software tool. So, CORAL architecture and features are presented as well. Optimal/predictive control results of the Riera Blanca catchment of Barcelona network using the CORAL software are presented in the results section. Finally, conclusions close the paper.

\section{MODELLING PRINCIPLES}

Complex non-linear rainfall-runoff models are very useful for off-line operations (calibration and simulation) of the sewerage network, but for on-line computation purposes, such as the global optimal control, a simple model structure must be selected with the following features (Norreys \& Cluckie 1997; Cluckie et al. 1999):

- Representativeness of the main network behaviours: it provides an evaluation of the hydrological and hydraulic variables of the network and its response to rain inputs and control actions at the actuators (gates, pumps, etc). In particular, it guarantees the mass balance and the stability of the system.

- Simplicity, expandability, flexibility and speed: It uses a simplest approach capable of achieving our purpose, the preferred analysis allows the modelled portion of the sewer system to be expanded and/or modified.

- Amenability to on-line calibration and optimization: the modelling approach is supported by theoretical knowhow and algorithms that have been well tested in the field of automatic control.

One possible model methodology to derive a rainfallrunoff real-time model of a sewerage network is through a simplified graph relating the main sewers and set of virtual and real reservoirs (Cembrano et al. 2004). A virtual reservoir is an aggregation of a catchment of the sewer network which approximates the hydraulics of rain, runoff and sewage water retention (Figure 1). 


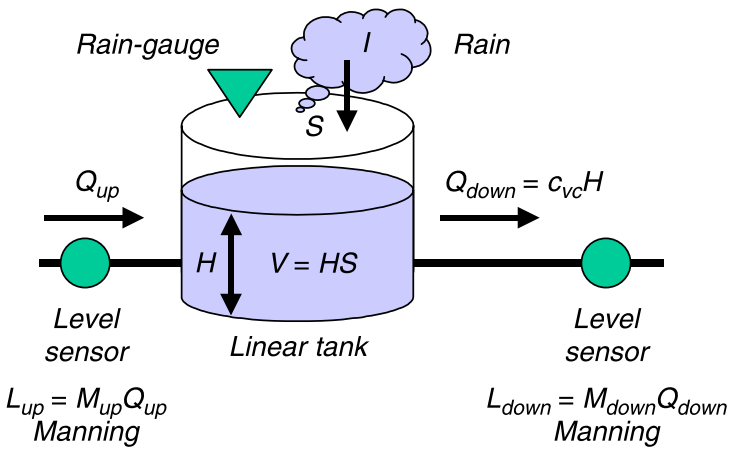

Figure 1 | Virtual reservoir model of a catchment.

The hydraulics of virtual reservoirs are:

$\frac{\mathrm{d} V(t)}{\mathrm{d} t}=Q_{\mathrm{up}}(t)-Q_{\mathrm{down}}(t)+\varepsilon S I(t)$

where: $V$ is the volume of water accumulated in the catchment, $Q_{\text {up }}$ and $Q_{\text {down }}$ are flows entering and exiting the catchment, $I$ is the rain intensity falling in the catchment, $S$ its surface and $\varepsilon$ the absorption coefficient.

Output sewer flow $Q_{\text {down }}$ can be related with the volume of water accumulated in the catchment $V$ assuming a linear relation by:

$Q_{\text {down }}(t)=c_{\mathrm{vc}} V(t)$

where $c_{\mathrm{vc}}$ is the volume-flow conversion coefficient. Given a network topology, a discrete time version of its model using modelling Equations (1) and (2) is:

$$
\begin{aligned}
V(k+1) & =V(k)+\left(Q_{\mathrm{up}}(k)-c_{\mathrm{vc}} V(k)+S I(k)\right) \Delta t \\
& =\left(1-c_{\mathrm{vc}} \Delta t\right) V(k)+\varepsilon S I(k) \Delta t+Q_{\mathrm{up}}(k) \Delta t
\end{aligned}
$$

(a)

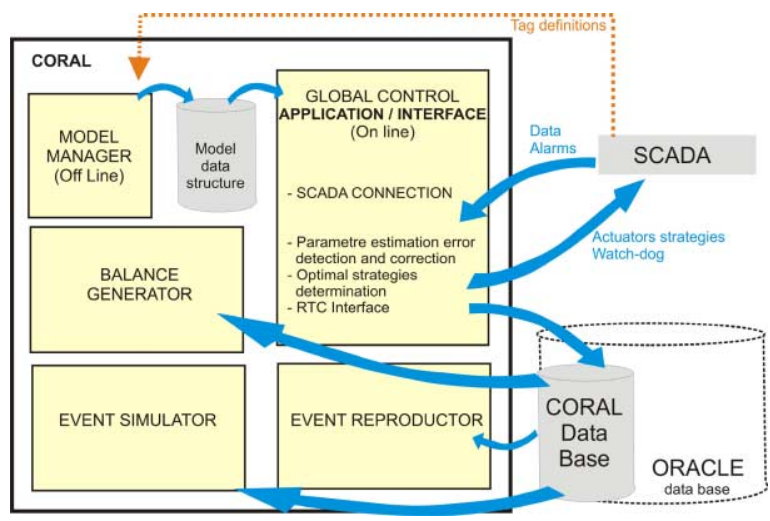

(b)

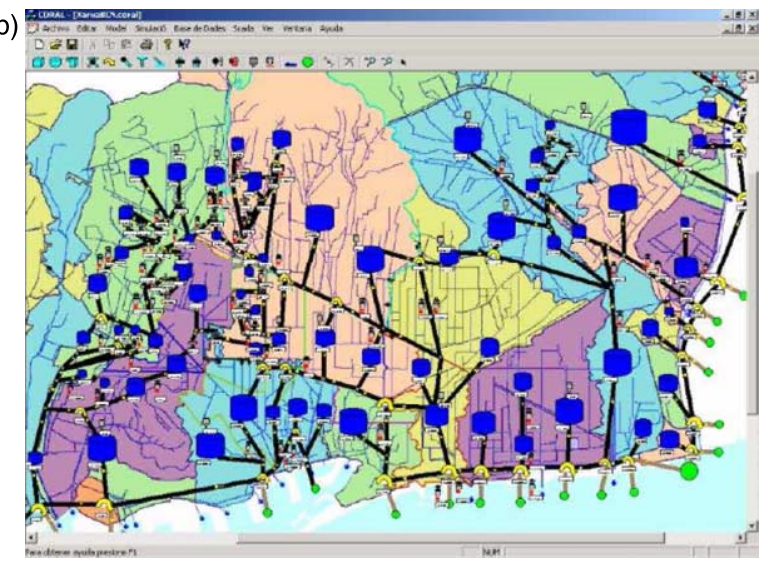

where $V$ is a vector of the virtual reservoir volumes, $S I$ is a vector corresponding to the addition of the products of areas by raining intensities, $Q_{\text {up }}$ is a vector containing the upstream water levels and $Q_{\text {down }}$ is a vector containing the downstream water levels.

Using this modelling methodology, a simplified model of an urban drainage network is created. Its structure depends on the topology of the network. Parameter $S$ must be supplied according to geographic characteristics of each catchment and parameters $\varepsilon$ and $c_{\mathrm{vc}}$ must be estimated for each catchment using real data from the sensors in the network. Off-line parameter estimation is carried out using historic data. On-line parameter estimation uses adaptive recursive identification techniques to update the parameter values at each time step using new readings of the telemetry system (Ljung 1999).

\section{PREDICTIVE/OPTIMAL CONTROL OF SEWER NETWORKS}

\section{Model predictive control (MPC) algorithm}

The optimal control goals in urban drainage systems are generally concerned with environmental protection, in particular, flooding prevention and minimization of CSO. The objective of applying optimal control is to compute, ahead of time, feasible strategies for the actuators in the network which produce the best admissible states of the network, in terms of these objectives, during a certain horizon.

Figure 2 | CORAL software: (a) CORAL Architecture. (b) CORAL model manager. 
The control period must be defined taking into account the telemetry system sampling time and the time constants of the actuators in the network. The optimization horizon must be selected considering the hydraulic time constants of storm water evacuation, reservoir depletion and/or other factors affecting the urban drainage management.

The computation of the optimal control set-points to be applied at the actuators is based on predictive control (Camacho \& Bordons 1999; Maciejowski 200I). More precisely, it applies the receding horizon strategy, based on determining a virtual control input sequence of present and future values $\left(u_{k}, u_{k+1} \ldots u_{k+N}-1\right)$ that optimizes an open-loop performance function, according to a prediction of the system evolution over the horizon $N$. This prediction is performed assuming that disturbances (rain measures) and model parameters will keep constant during the horizon. However, only the first control input of sequence $\left(u_{k}\right)$ is actually applied to the system, until another sequence based on more recent data is computed as shown in Figure 3. Predictive Control strategies have been applied previously in sewers systems by (Gelormino \& Ricker 1994).

\section{Multicriteria optimization}

The cost function is the mathematical expression of the urban drainage management goals. In the Barcelona case, flood prevention is the first priority. Secondly, CSO reduction must be sought, albeit without compromising flood prevention and, finally, the network must drain as much water as possible, provided this does not interfere with any of the first two objectives. This type of problems is usually classified as multi-objective optimization.

One of the most well known multi-objective techniques is the linearly weighted sum, where the vector objective function is scaled in such a way that the value judgment of the decision making can be incorporated:

$\min _{x} \sum_{i=1}^{r} w_{i} f_{i}(x)$

subject to : $x \in \chi$

where $w_{i}$ are the different scaling weights and $\chi$ is the feasible solution set.
For the CORAL optimal control problem description, a multi-objective cost function is chosen as the summation over the optimization horizon of a weighted sum of the following terms:

- a sum of overflows in the sewers (one for each sewer in the network),

- a sum of overflow volumes to the sea at each time interval and the instantaneous volume in real reservoirs (detention tanks)

- a sum of the deviations of the WWTPs from the maximum flow

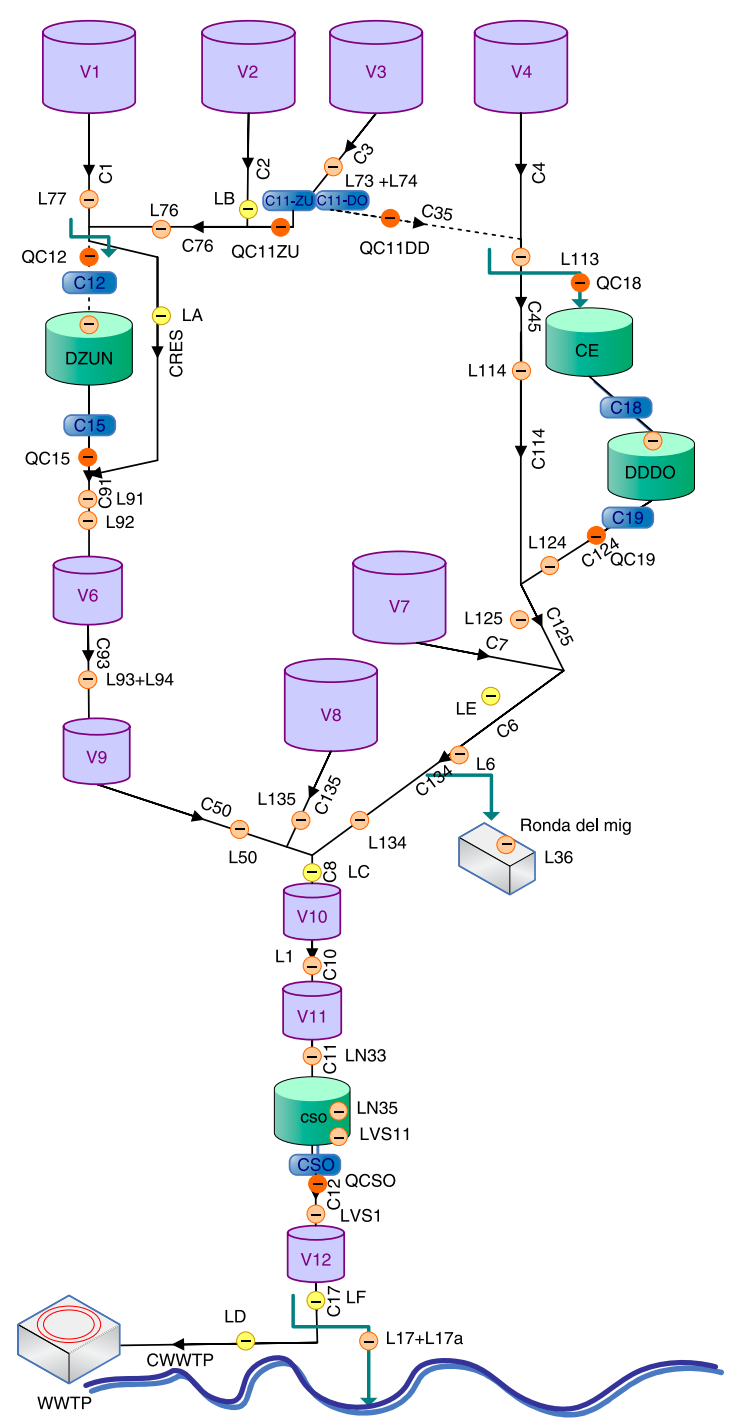

Figure 3 | Riera Blanca Catchment Conceptual Model using CORAL modelling methodology. 
The expression of the cost function is as follows:

$$
\begin{aligned}
J= & \sum_{k-0}^{N-1}\left(\alpha J_{\text {flood }}(k)+\beta J_{\mathrm{CSO}}(k)+\gamma J_{\mathrm{WWTP}}(k)\right) \\
= & \sum_{k-0}^{N-1}\left(\alpha \sum_{i} \max \left(0,\left(q_{i}(k)-q_{i}^{*}\right)\right)+\beta \sum_{j} \operatorname{CSO}_{j}(k)\right. \\
& \left.+\gamma \sum_{l}\left|q_{\mathrm{WWTP}, l}(k)-q_{\mathrm{WWTP}, l}^{*}\right|\right)
\end{aligned}
$$

where: $N$ is the optimization horizon using by the controller in number of sampling periods, $k$ indicates the time, $q_{i}$ is the flow through sewer $i$ at time $k, q_{j}^{\prime \prime}$ is the maximum allowed flow through sewer $j, \mathrm{CSO}_{j}^{k}$ is the combined sewer overflow volume of site $j$ at time $k, q_{\mathrm{WWTP}, l}$ is the flow treated by WWTP plant $l$ and $q_{\mathrm{WWTP}, l}^{*}$ is the maximum flow that can treat. Finally, $\alpha, \beta$ and $\gamma$ are the weights associated to the different control objectives.

The weighting factor of each objective is adjusted to guarantee that the flood prevention goal takes priority over the other two.

The optimization must be performed with respect to the control variables $u_{k}$ (set-points of the gate local controllers) and it is subject to a set of constraints, namely:

- linear equality equations expressing the mass balance in reservoirs (real and virtual)

$x_{k+1}=A x_{k}+B_{u} u_{k}+B_{w} w_{k}$

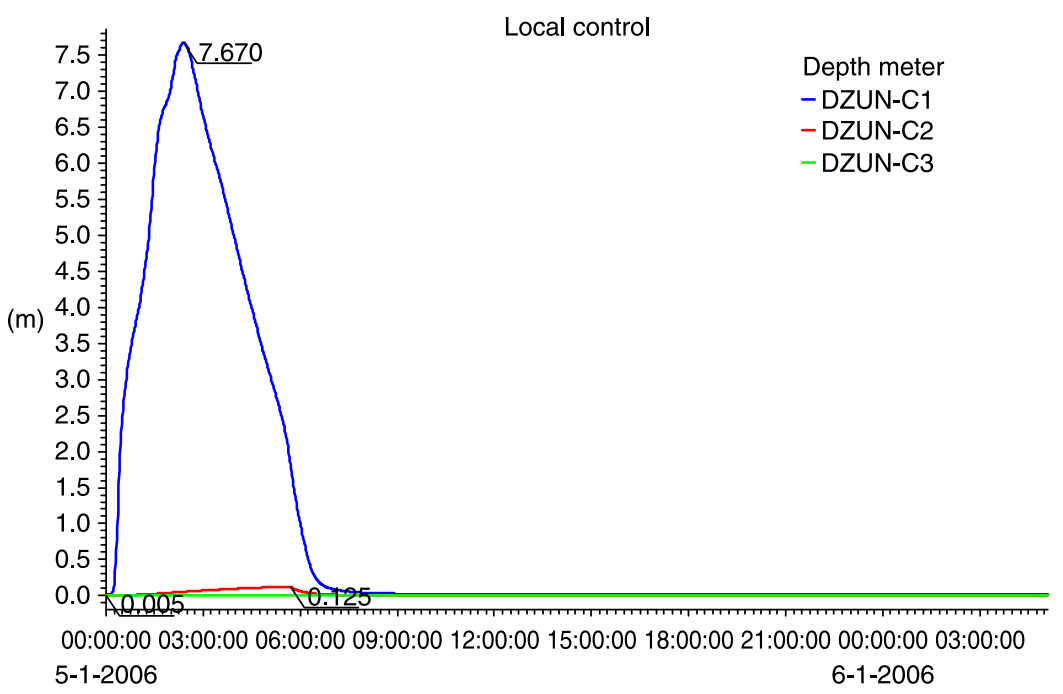

where $x_{k}$ are the state variables (reservoir volumes) and $w_{k}$ is the disturbance (rain)

- non-linear equality flow equations related to the overflow devices

$g\left(x_{k}, u_{k}\right)=0$

- and bound constraints on the operative range of gates, on the allowable flows through the sewers and on maximum reservoir capacities

$$
\begin{aligned}
& x_{\min } \leq x \leq x_{\max } \\
& u_{\min } \leq u \leq u_{\max }
\end{aligned}
$$

\section{CORAL SOFTWARE FEATURES AND ARCHITECTURE}

The a global predictive/optimal control system of Riera Blanca is implemented using a software tool named CORAL.

CORAL's architecture is presented in Figure 2(a). CORAL is composed of:

- a model manager which allows the user to create, modify or update an operational model of the network to compute the effect of control actions on the future state of the network (see Figure 2(b)).

- an automatic equation generation module which produces a set of equations describing the problem

- an on-line optimization procedure to select set point for the actuators to achieve operational goals

Figure 4 | DZUN in local control. 
- a real-time predictive control scheme which may be connected to a SCADA to incorporate real measurements and to produce set points to be sent to actuators, in general after confirmation by an operator

- an on-line recursive parameter estimation module to adapt model parameters with the latest readings from the SCADA

- a data base (ACCESS or ORACLE)

- a robust commercial optimization solver (GAMS 1997).

CORAL has been jointly developed by CLABSA and UPC. CORAL was already successfully tested off-line in Escola Industrial Barcelona test catchment as described in (Cembrano et al. 2004). CORAL was also tested on the Murcia (Spain) network. In this last city, it was used to do a study of feasibility and potential benefits of the construction of 3 detention tanks and the use of diversion gates. This paper deals with the demonstration of the on-line use of CORAL for predictive optimal control in the Riera Blanca catchment of the Barcelona network. This catchment has been simulated using a high fidelity model using MOUSE. CORAL interfaces with MOUSE interchanging readings from the limnimeters and sending the control commands to the gates as if it was connected with the real SCADA system.

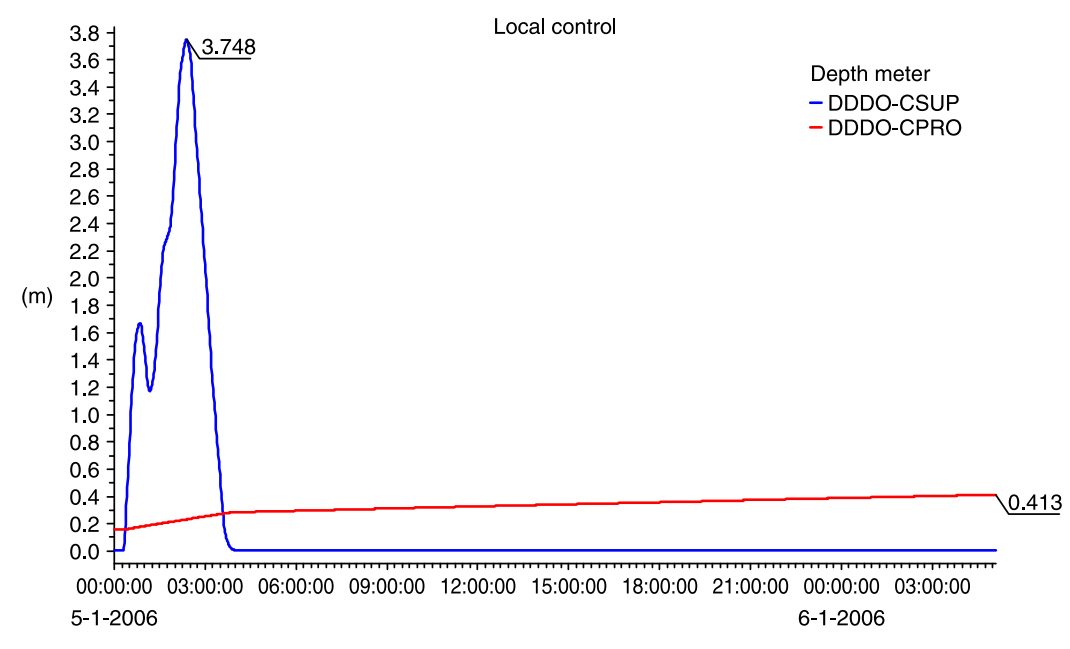

Figure 5 | DDDO in local control.

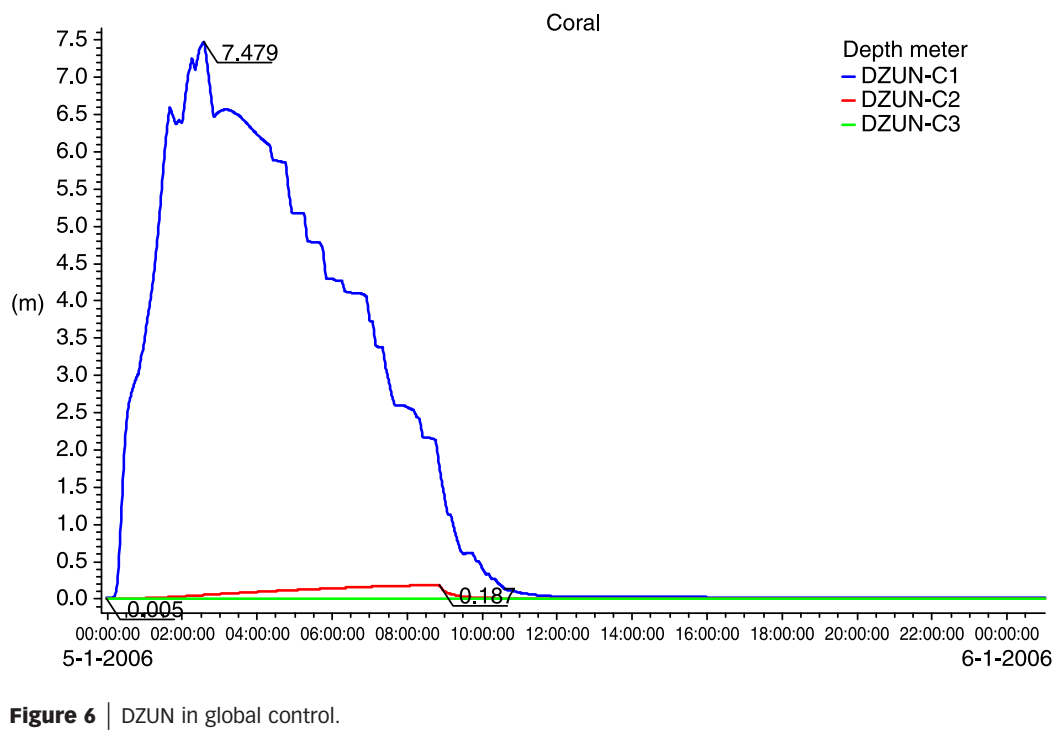




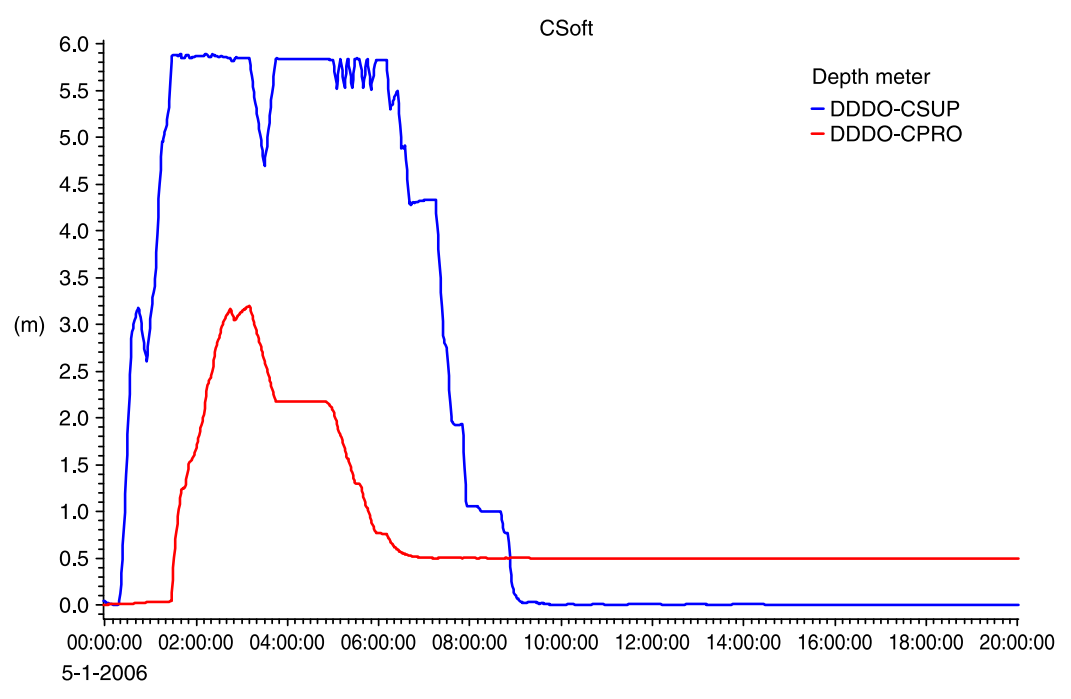

Figure 7 | DDDO in global control.

\section{RESULTS IN THE RIERA BLANCA CATCHMENT TEST}

\section{Catchment description}

The Riera Blanca pilot catchment is presented in Figure 3. This catchment has two detention tanks:

- Zona Universitaria Detention Tank (DZUN)

- Doctors Dolsa Detention Tank (DDDO) and a sewer that will be used for in-line detention: CSO sewer (CSO)

The gates that will be operating in global control are:

- retention gates at the output of detention tanks (C15 and C19) and inline retention sewer (CSO)

- divertion gate (C11) that connects the two detention tanks.

- The in-line retention gate CSO-gate

Two flooding control points have been established:

- L50 at a distant point of Zona Universitaria Detention Tank

- L6 at a distant point of Doctors Dolsa Detention Tank

Eleven measurement points are considered and a Waste Water Treatment Plant at the end of this catchment with a treatment capacity of $2 \mathrm{~m}^{3} / \mathrm{s}$. Flows sent to this plant bigger than this value will be released to the sea generating CSO.

The operative goals of this catchment are the following in order of priority:
- minimize (if possible avoid) flooding in any part of the catchment under the control of the detention tanks

- minimize CSO to the sea

- maximize sewage treatment at the WWTP

\section{Results on a real scenario}

This scenario corresponds to a common winter rain occurred in Barcelona (approx. a 2-month return period). It is neither very intense nor very long. The maximum intensity occurs in rain gauge $\mathrm{P} 11$, with $49.2 \mathrm{~mm} / \mathrm{h}$, and the duration is 3 hours. The main purpose of this event is to reduce $\mathrm{CSO}$ overflow since this event has no problems of flooding. The gate located on the top of the catchment, C11, is a flow diversion gate. Using local management, this gate is only operating in case DZUN is completely full. The first thing observed is that, in global control this gate is used much more. Moreover, the dewatering time is larger in

Table 1 | CSO results

\begin{tabular}{lll} 
Local control & Global control & cso variation (\%) \\
\hline $203,353 \mathrm{~m}^{3}$ & $167,224 \mathrm{~m}^{3}$ & $-18 \%$ \\
\hline
\end{tabular}

Table 2 | WWTP results

Local control

Global control

WWTP variation (\%)

$77,208 \mathrm{~m}^{3}$

$115,863 \mathrm{~m}^{3}$

$50 \%$ 
global control than in local control because CORAL is reducing the $\mathrm{CSO}$. The following pictures correspond to the volume evolution during the scenario in Zona Universitaria and Doctors Dolsa detention tanks respectively in local control (Figures 4 and 5) and in global control (Figures 6 and 7). Regarding the graphs more water is sent to Doctor Dolsa detention tank in the case of the Global Control. The reason is that CORAL attempts to balance the water distribution between the different parts of the network and, in this case, it detects that DDDO have less water than DZUN.
Looking at the WWTP and CSO results, it is clear that CORAL control outperforms the results of the simple control; the $\mathrm{CSO}$ reduction as compared to the simple control is 18\% and the WWTP increases a 50\% (see Tables 1 and 2).

Moreover, looking at the Figure 8, it can be noticed that the WWTP is used for longer time (global control in red colour versus local control blue colour, and in Figure 9 show the comparison of CSO volumes. Clearly, the global management reduces significantly the volume of CSO in this scenario. It can be noticed that the WWTP operational

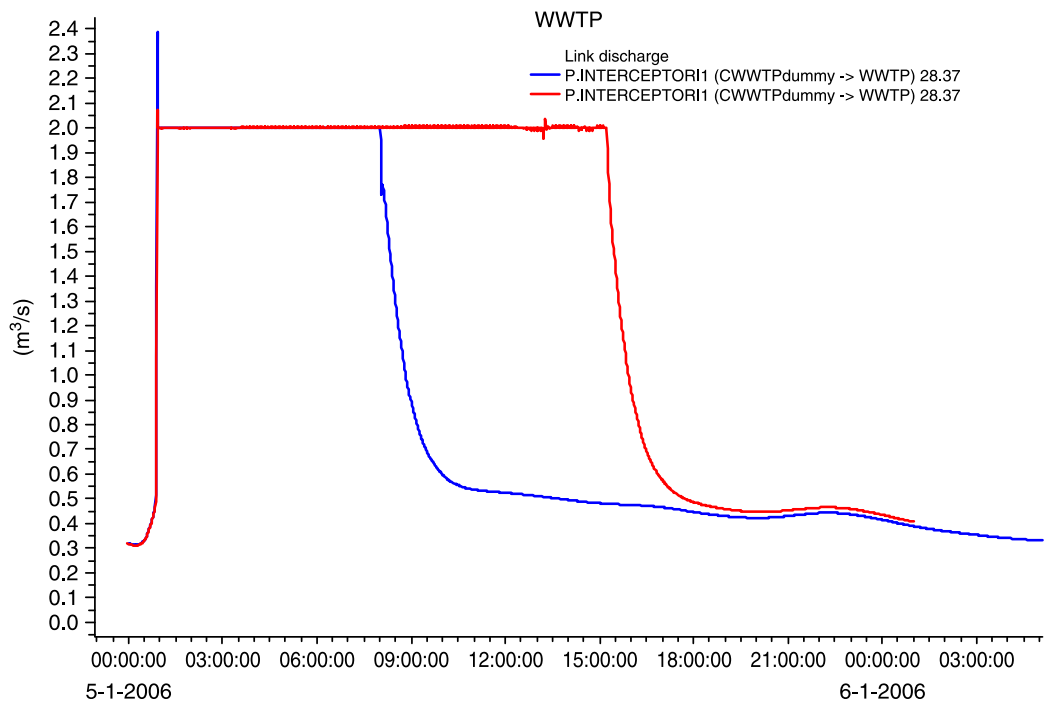

Figure 8 | WWTP volume in local (blue/dark grey) and global control (in red/light grey).

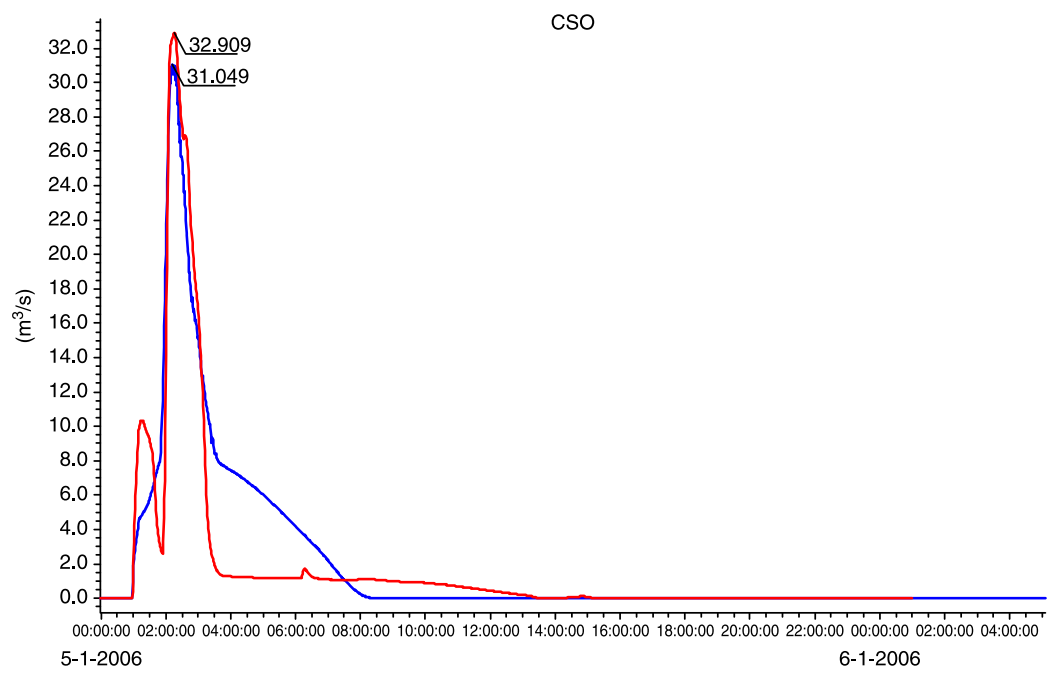

Figure 9 | CSO volume to the Sea in local (blue/dark grey) and global control (in red/light grey). 


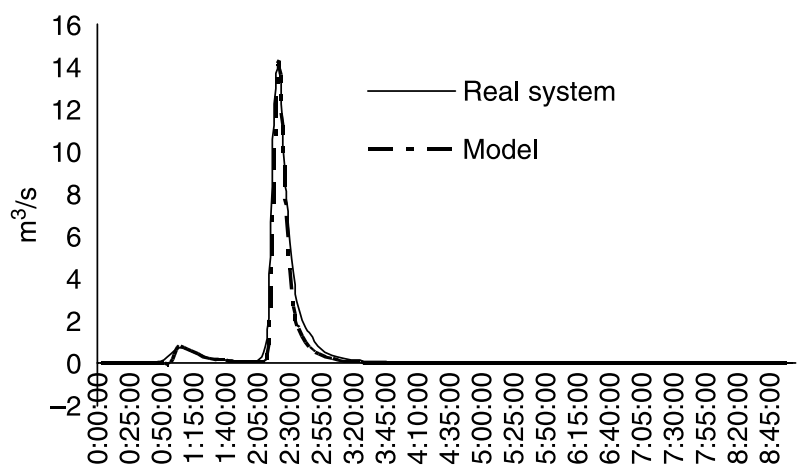

Figure 10 Comparison of the predicted and real flow at the output of virtual tank 3.

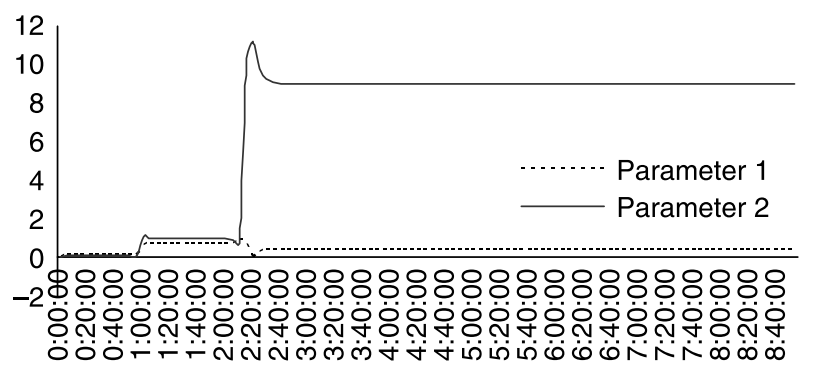

Figure 11 | Parameter autocalibration of virtual tank 3.

constraints are satisfied when the global control is used since they are imposed as constraints in the MPC optimization problem.

Finally, Figure 10 shows the flow predicted by the control model at the output of virtual tank 3 compared with the real flow, while Figure 11 shows the recursive parameter estimation of the two parameters of virtual model corresponding to this virtual tank using classical recursive least squares algorithms (Ljung 1999).

\section{CONCLUSIONS}

The proposed global predictive control of sewer complex networks (CORAL) has the capabilities to build a real time control model of the proposed test catchments, using the existing components of the CORAL library and including correctly all the elements to be controlled. All the components of a complex network, such as the case of Barcelona sewer network, have been modelled in CORAL: virtual reservoirs of the simple catchments, level-flow translation in sewers, level-volume translation in detention tanks, rain-forecast, in-line retention. And one important feature of CORAL model is his capacity to auto-calibrate the parameters of the model. And CORAL has been connected successfully with a simulator of the Barcelona sewer network named MOUSE. Regarding the final results obtained in the tests, they are very promising: Using one comprehensive objective function, important improvements are achieved simultaneously in CSO minimization, flooding prevention and efficient WWTP usage, as compared to the application the simple local control currently in use at CLABSA. The results of this project contribute to proving that CORAL is a general tool that can be applied to any complex sewer networks, such as the case of Barcelona city.

\section{ACKNOWLEDGEMENTS}

This research project, partially funded by $\mathrm{R}+$ Di Alliance, has been carried out jointly by CLABSA (leader of the project), UPC and CSIC. The first four authors are members of the SAC (Advanced Control Systems) Group of UPC. Research in this group is partially supported by DGR de la Generalitat de Catalunya under contract GEPREDO 2006ITT-10001and grant SAC 2005SGR00537 and by the Spanish Ministry of Science and Education under grant ITACA DPI-2006-11944.

\section{REFERENCES}

Brdys, M. A. \& Ulanicki, B. 1994 Operational Control of Water Systems: Structures, Algorithms and Applications. Prentice Hall International, UK.

Camacho, E. F. \& Bordons, C. 1999 Model Predictive Control in Advanced Textbooks in Control and Signal Processing. Springer, London.

Cembrano, G., Quevedo, J., Salamero, M., Puig, V., Figueras, J. \& Martí, J. 2004 Optimal control of urban drainage systems. A case study. Control Eng. Pract. 12(1), 1-9.

Cembrano, G., Quevedo, J., Puig, V., Pérez, R. \& Figueras, J. 2005 First results of predictive control application on water supply and distribution in Santiago-Chile. Proceedings of the IFAC'05 World Congress, Prague.

Cluckie, I. D., Lane, A. \& Yuan, J. 1999 Modelling large urban drainage systems in real time. Water Sci. Technol. 39(4), 21-28.

GAMS 1997 The Solver Manuals. GAMS Development Corporation, Washington, DC. 
Gelormino, M. S. \& Ricker, N. L. 1994 Model-predictive control of a combined sewer system. Int. J. Control 59, $793-816$

Ljung, L. 1999 System Identification: Theory for the User. Prentice Hall. Maciejowski, J. 20or Predictive Control with Constraints. AddisonWesley, Wokingham, UK.

Norreys, R. \& Cluckie, I. 1997 A novel approach to real-time modelling of large urban drainage systems. Water Sci. Technol. 36(8-9), 19-24.

Ocampo-Martínez, C., Ingimundarson, A., Puig, V. \& Quevedo, J. 2008 Objective prioritization using lexicographic minimizers for MPC of sewer networks. IEEE Trans. Control Syst. Technol. 16(1), 113-121.

Pleau, M., Colas, H., Lavallée, P., Pelletier, G. \& Bonin, R. 2005 Global optimal real-time control of the Quebec urban drainage system. Environ. Model. Softw. 20, 401-413.

Price, R. 2000 Hydroinformatics and urban drainage: an agenda for the 21st century. J. Hydroinform. 2(2), 133-147.

Schütze, M., Campisano, A., Colas, H., Schilling, W. \& Vanrollegheme, P. A. 2004 Real-time control of urban wastewater systems-where do we stand today? J. Hydrol. 299(4), 335-348. 